DOI: https://doi.org/10.11144/Javeriana.umed61-4.disf

\title{
Disfagia en el adulto mayor
} Dysphagia in the Elderly

Recibido: 01 Junio 2020 | Aceptado: 10 Julio 2020

\section{Carlos José Ariza-Galindo}

Unidad de Geriatría, Hospital Universitario

San Ignacio, Bogotá, Colombia. Instituto de Envejecimiento, Pontificia Universidad Javeriana, Bogotá, Colombia. Facultad de Medicina, Pontificia

Universidad Javeriana, Colombia

Diana María Rojas Aguilar

Unidad de Geriatría, Hospital Universitario

San Ignacio, Bogotá, Colombia. Instituto de

Envejecimiento, Pontificia Universidad Javeriana,

Bogotá, Colombia. Facultad de Medicina, Pontificia

Universidad Javeriana, Colombia

a Autor de correspondencia: arizacarlos@javeriana.edu.co

Cómo citar: Ariza-Galindo CJ, Rojas Aguilar DM. Disfagia en el adulto mayor. Univ. Med. 2020;61(4). h ttps://doi.org/10.11144/Javeriana.umed61-4.disf

\section{RESUMEN}

La disfagia orofaríngea se define como la dificultad para el paso del bolo alimentario desde la boca hasta el esófago inferior. Es considerado un nuevo síndrome geriátrico común en la población anciana. Su prevalencia aumenta con la edad y plantea problemas especiales en este grupo de pacientes, pues compromete su estado nutricional, al aumentar el riesgo de neumonía de etiología aspirativa y afecta su calidad de vida. Ha llegado a describirse como un nuevo síndrome geriátrico y una pandemia emergente, por lo que se considera uno de los principales retos de la medicina geriátrica. Dentro de esta revisión temática, se intentará definir la disfagia en el adulto mayor, su clasificación, fisiopatología y tratamiento, así como su presentación en condiciones especiales como la demencia.

\section{Palabras clave}

envejecimiento; trastornos de la deglución; demencia.

\begin{abstract}
Oropharyngeal dysphagia is defined as the difficulty for the passage of the food bolus from the mouth to the lower esophagus. It is considered a new common geriatric syndrome in the elderly population. Its prevalence increases with age and poses special problems in this group of patients, compromising nutritional status, increasing the risk of pneumonia of aspirational etiology, and affecting quality of life. It has come to be described as a new geriatric syndrome and an emerging pandemic, which is why it is considered one of the main challenges of geriatric medicine. Within this thematic review we will try to define oropharyngeal dysphagia in the elderly, classification, pathophysiology, and treatment, as well as its presentation in special conditions such as dementia.

Keywords

aging; deglutition disorders; dementia.
\end{abstract}




\section{Introducción}

La disfagia se está volviendo cada vez más común en las personas que envejecen y, como los síndromes geriátricos clásicos, implican un deterioro funcional relevante. La prevalencia de disfagia es más elevada en pacientes de edad avanzada con trastornos neurológicos, sobre todo en pacientes con accidente cerebrovascular, demencia y enfermedad de Parkinson (1).

El envejecimiento es un proceso asociado a una serie de cambios significativos en los nervios y en la función muscular. En los ancianos, dichos cambios pueden predisponer a disfagia, como pérdida de fuerza de la mandíbula, disminución de producción de saliva, alteraciones en la dentición, así como un aumento del tejido conectivo y graso en la lengua, que afectan la fase oral de la deglución.

Sin embargo, típicamente, los cambios relacionados con la edad en esta fase de la deglución no resultan en disfagia (2). Existe disminución de la percepción somatosensorial; de la densidad y actividad muscular prolongada de los músculos masticatorios, maseteros y pterigoideo medial; de la actividad muscular en los constrictores de la faringe, y del tono del esfínter esofágico superior. Después de los 60 años de edad, la deglución faríngea es significativamente más larga y, a veces, requiere múltiples tragos por bolo, lo cual puede aumentar el riesgo de aspiración (2).

Una de las principales causas de disfagia orofaríngea corresponde a cambios neuromusculares, las cuales pueden ser más prevalentes durante el envejecimiento; sin embargo, en pacientes con trastornos neurocognitivos (enfermedad de Alzheimer, demencia de origen vascular, demencia por enfermedad de Parkinson, etc.) pueden presentarse, incluso, desde estadios iniciales y con mayor impacto en su calidad de vida (3).

Por ende, en la población que envejece puede, con frecuencia, conducir a desnutrición severa, broncoaspiración, neumonía y muerte $(4,5)$. La prevalencia descrita para disfagia es variable; pero se calcula una media de disfagia para ancianos que viven en comunidad del $15 \%$ y hasta del $30 \%$ para los ancianos ingresados al hospital (6). Otros autores reportan una prevalencia de disfagia del $10 \%$ para ancianos de la comunidad y una prevalencia del $50 \%$ para ancianos residentes de hogares geriátricos; otros, por el contrario, documentan una prevalencia en personas mayores de 65 años entre el $7 \%$ y el 22 $\%(7,8)$. En Colombia, un estudio realizado por Frías Ordóñez et al. (9), donde se estudiaban las características de pacientes no hospitalizados en un centro especializado en gastroenterología, se encontró una baja prevalencia de disfagia $(2,7$ $\%)$; sin embargo, en este estudio también se incluyeron pacientes jóvenes.

Entre el $27 \%$ y el $50 \%$ de los pacientes con accidente cerebrovascular presentan disfagia (10) y, a su vez, mayor riesgo de discapacidad grave o muerte $(11,12)$. Dentro de los trastornos neurocognitivos con mayor presencia de disfagia se encuentran la demencia frontotemporal (fases avanzadas), la demencia por enfermedad de Alzheimer (fases iniciales y tardías), la demencia de origen vascular, la demencia por cuerpos de Lewy y la demencia por enfermedad de Parkinson; además, la parálisis supranuclear progresiva, que afecta todas las fases de la deglución $(13,14,15,16)$.

\section{Clasificación}

En cuanto a la ubicación fisiopatológica o fase alterada puede clasificarse como:

Disfagia orofaríngea: denominada también disfagia de transferencia, en la que el signo principal es la dificultad para iniciar la deglución, necesidad de intentos repetidos para deglutir, regurgitación nasal durante la deglución y tos o ahogo inmediatamente después de intentar tragar. Durante este proceso existe dificultad para la formación del bolo dentro de la cavidad oral, lo que provoca retención en la boca y derrame del bolo a través de los labios, acompañado de sialorrea. La retención del bolo puede producirse en la faringe, secundario a problemas de propulsión lingual, faríngea o alteración en la apertura del esfínter esofágico superior (17). 
Disfagia esofágica: es la incapacidad para el paso de los alimentos a través del esófago una vez que los alimentos han pasado con éxito al esófago proximal. Esta alteración, generalmente, se produce por trastornos de la propulsión, afectación peristáltica, lesiones circunferenciales o inhibición de la deglución esofágica, por compromiso de la musculatura lisa del esófago torácico y parte del esófago cervical $(17,18)$.

\section{Fisiopatología}

La disfagia orofaríngea es el resultado de múltiples factores y causas (tabla 1). Algunas etiologías se dividen en una o más categorías: trastornos neurológicos, como enfermedades, lesiones o anomalías del sistema nervioso central, sistema nervioso periférico o unión neuromuscular; factores físicos o estructurales relacionados con deformidades estructurales de la cabeza y el cuello, especialmente después de cirugías para cáncer de orofaringe (glosectomía), y causas inmunológicas, como miopatía inflamatoria. Algunos medicamentos se han asociado con debilidad muscular, sarcopenia y disfagia, incluidos los fármacos psicótropos, benzodiacepinas, antinflamatorios y algunos medicamentos vasoactivos (19).

\section{Tabla 1}

Causas de la disfagia orofaríngea

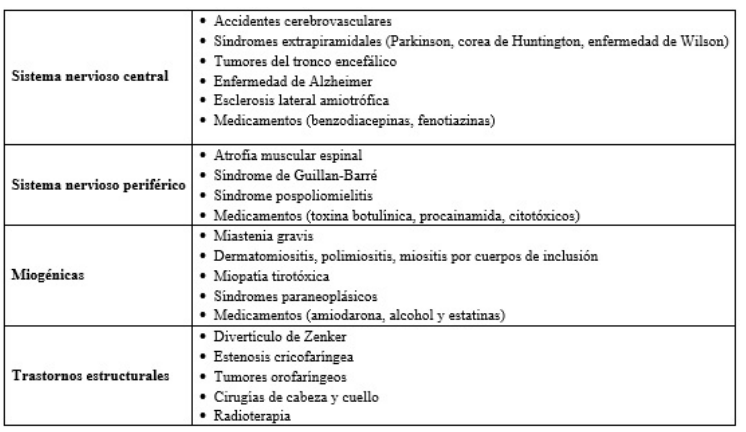

Fuente: adaptado de Cook IJ. Oropharyngeal dysphagia. Gastroenterol Clin

North Am. 2009 Sep;38(3):411-31

Diversas revisiones han descrito como principales factores de riesgo para disfagia orofaríngea: edad, baja capacidad funcional, presencia de enfermedades neurodegenerativas y uso de medicamentos con acción sobre el sistema nervioso central y depresión $(20,21)$.

\section{Diagnóstico}

La disfagia en los ancianos se malinterpreta como una parte normal del envejecimiento tanto por el personal médico como por los propios pacientes, razón por la cual no se detecta de forma precoz. Por esta razón, se debe contemplarse que con el envejecimiento puede surgir una mayor carga de enfermedad y un mayor consumo de medicamentos que hace más vulnerables a los ancianos y, en ciertas condiciones, generar alteraciones en la deglución. Por ende, la intervención diagnóstica de la disfagia en los pacientes ancianos siempre debe tener presente estas singularidades, al igual que los cambios fisiológicos mencionados. En el momento de diagnosticar la disfagia es importante realizar un adecuado interrogatorio que establezca la temporalidad de los síntomas o si presenta un carácter progresivo o intermitente; identificar su asociación con otros síntomas, como pérdida de peso o sangrado; establecer las consistencias con las cuales percibe dificultad del paso del bolo alimenticio y su asociación o no con odinofagia. En pacientes con disfagia, se recomienda interrogar por los síntomas y llevar a cabo un examen físico dirigido, como evaluación inicial para identificar pacientes con disfagia orofaríngea. GRADE modificado: 1C. Recomendación fuerte, evidencia de muy baja calidad (22).

El objetivo de evaluar la función de la deglución es determinar si el individuo tiene disfagia, qué función fisiológica está trastornada y si un cambio en la forma o consistencia en la que se toma la nutrición sería seguro y beneficioso para la persona (23). En pacientes con disfagia se recomienda la identificación rápida de las personas con disfagia orofaríngea, debido al mayor riesgo de aspiración. GRADE modificado: 1C. Recomendación fuerte, evidencia de baja calidad (22).

Se puede realizar el cribado clínico junto a la cama, para detectar un posible problema de 
deglución y determinar la idoneidad de una evaluación instrumental. El clínico observa cómo el paciente coloca un bolo líquido o sólido en la boca, qué tan eficientemente está contenido el bolo en la fase oral y la cantidad de tiempo necesario para mover el bolo por la vía oral $y$, en última instancia, la cantidad de tiempo desde la fase oral hasta la deglución (24). El test volumen-viscosidad de deglución (25) es una prueba clínica validada con propiedades psicométricas óptimas que se puede realizar junto a la cama de los pacientes (26), y permite seleccionar el mejor volumen y viscosidad de los alimentos para evitar penetraciones en la vía aérea. Durante la valoración, el paciente recibe diferentes volúmenes de alimento $(5 \mathrm{ml}, 10 \mathrm{ml}$ o $20 \mathrm{ml}$ ) y diferentes viscosidades (líquido, néctar o pudín), a fin de verificar durante la prueba la eficacia y seguridad del procedimiento (27).

En una revisión sistemática se analizaron diversas pruebas clínicas y se identificaron cuatro con una sensibilidad mayor del $70 \%$ y una especificidad mayor del $60 \%$ : prueba de detección de Toronto, prueba de deglución con volumen-viscosidad, prueba de deglución de 3 onzas de agua y prueba de la tos (26), las cuales en pacientes neurológicos han demostrado utilidad en la identificación de disfagia orofaríngea y riesgo de aspiración (22).

\section{Evaluación instrumental para la disfagia orofaríngea}

Entre las herramientas para la evaluación instrumental de la disfagia orofaríngea, la más común es la videofluoroscopia. La evaluación instrumental provee información importante acerca de la naturaleza de la fisiología de la deglución y la disfunción que genera la disfagia, que permite el tratamiento adecuado y la prevención de los episodios de aspiración (28):

\section{Videofluoroscopia}

Es la técnica más específica para el estudio de las alteraciones fisiopatológicas de la deglución en ancianos con disfagia. Los principales signos encontrados son el fallo en el sellado labial y la incapacidad para formar el bolo, que ocasionan pérdida de este. El residuo faríngeo es el principal signo en videofluoroscopia de cambios en la eficacia de la fase faríngea. El signo principal en la alteración de la seguridad durante la fase oral es la modificación del sello glosopalatino (lenguapaladar blando), lo que da lugar a la caída del bolo a la hipofaringe antes de que se produzca la activación de la respuesta motora orofaríngea y mientras la vía aérea permanece abierta. Los signos en videofluoroscopia son las penetraciones en el vestíbulo laríngeo y las aspiraciones a la vía aérea. La penetración laríngea se produce cuando el bolo entra en el vestíbulo laríngeo, y la aspiración, cuando el bolo atraviesa las cuerdas vocales verdaderas y entra en la vía aérea (29).

Evaluación endoscópica por fibra óptica de la deglución

Es un procedimiento común y estándar utilizado para evaluar pacientes con disfagia orofaríngea. A menudo, se compara con el procedimiento fluoroscópico, que se usa con el mismo propósito, pero tiene una perspectiva muy diferente de la anatomía. Primero, los dos procedimientos se complementan entre sí, de muchas maneras. Cada uno de ellos se usa con pacientes ancianos para determinar si presentan alteraciones en la deglución, y si está presente, definir cuál es el patrón o la naturaleza de esta alteración. En segundo lugar, cada uno de ellos se utiliza con el propósito de evaluar estrategias de comportamiento o alteraciones del bolo para ver su efecto en la deglución, es decir, se consideran evaluaciones terapéuticas (30).

La evaluación endoscópica por fibra óptica de la deglución incluye dos partes: en la primera hay una evaluación preliminar de la anatomía en relación con la deglución, las secreciones en la hipofaringe y la laringe, el movimiento de las estructuras clave evaluadas en tareas sin deglución, incluida la retracción de la base de la lengua, la elevación laríngea/ aritenoidea, la compresión de la pared faríngea cierre velofaríngeo, movilidad de las cuerdas 
vocales y cierre de la glotis. Durante la deglución, también se evalúa la retroflexión epiglótica y el cierre del espacio aéreo.

La segunda parte de la evaluación consiste en lograr que el paciente coma y beba varios líquidos y sólidos de diferentes tamaños y consistencias para observar directamente la seguridad y la eficacia de la deglución. Las variables para calificar incluyen: preparación oral del bolo (contención y eficiencia de la masticación), propulsión lingual del bolo e iniciación de la deglución (velocidad y tiempo con el flujo de bolo) y aclaración faríngea. Se observa el tiempo de retraso faríngeo en segundos y la ubicación del bolo al inicio de la deglución, la penetración y la aspiración $(31,32,33,34)$. La evaluación endoscópica por fibra óptica de la deglución tiene ventajas en comparación con la fluoroscopia, porque es un examen portátil y puede realizarse junto a la cama o en el hogar de residencia de los ancianos, sin necesidad de transportarlos a otro lugar; además, utiliza alimentos y líquidos reales sin bario agregado, y es un mejor examen terapéutico, porque se puede continuar por un periodo prolongado, lo que le da al examinador tiempo para probar estrategias múltiples, consistencias de bolo, etc.

En pacientes con disfagia esofágica se recomienda la endoscopia más el esofagograma con bario para mejorar el diagnóstico de enfermedad esofágica estructural. GRADE modificado: 1C. Recomendación fuerte, evidencia de muy baja calidad. Se recomienda un esofagograma cuando hay acceso local limitado para la realización de la endoscopia con la finalidad de evaluar lesiones estructurales significativas. GRADE modificado: 1C. Recomendación fuerte, evidencia de muy baja calidad (22).

\section{Tratamiento}

El tratamiento para la disfagia es sencillo, costoefectivo y si se aplica a tiempo, pueden evitarse un gran número de complicaciones que pueden tener un efecto negativo en la salud y la calidad de vida de los pacientes (35). El tratamiento para la disfagia orofaríngea en la población anciana se basa en medidas compensatorias, como adaptar fluidos con espesantes, con la finalidad de evitar problemas de seguridad, al igual que realizar posturas y maniobras, para compensar alteraciones biomecánicas $(27,36)$.

\section{Adaptación nutricional y de fluidos}

La adición de agentes espesantes a los fluidos para aumentar la viscosidad y para reducir el riesgo de penetración y aspiración es una práctica común en pacientes con disfagia orofaríngea, independientemente de su fenotipo (27). Varias publicaciones han demostrado la eficacia de este tratamiento en personas mayores con disfagia orofaríngea, al reducir la prevalencia de penetraciones del vestíbulo laríngeo y aspiración traqueobronquial $(37,38,39)$. Algunos estudios han demostrado mayor riesgo de deshidratación con el uso de espesantes, debido al mayor esfuerzo necesario para tragar y una menor adherencia a la nutrición, dadas las viscosidades mayores (40, 41, 42).

En cuanto a las indicaciones específicas para modificar consistencias, en el 2017 se revisaron y actualizaron las recomendaciones GRADE, por parte de la División de Investigación de Nutrición en Dinamarca. En ellas se plantea el uso de líquidos espesados solo después de una cuidadosa consideración, como estrategia compensatoria para facilitar la ingesta de líquidos, debido a que en los adultos con disfagia orofaríngea el efecto beneficioso sobre el resultado crítico es incierto y su uso parece tener una tendencia hacia la deshidratación y el rechazo del paciente (43). GRADE modificado 2B. Recomendación débil, evidencia de moderada calidad.

No se recomienda el uso de líquidos espesados con miel para lograr consistencias moderada/ extremadamente espesas como estrategia compensatoria para facilitar la ingesta de líquidos, ya que en adultos con disfagia orofaríngea el efecto beneficioso sobre el resultado crítico es incierto y su uso parece tener una tendencia hacia deshidratación, rechazo del 
paciente y pérdida de peso (43). Punto de buena práctica según consenso, no evidencia.

\section{Maniobras, estrategias posturales y praxias musculares}

Dos factores importantes durante el proceso de ingesta en los pacientes ancianos con disfagia orofaríngea son la verticalidad y la simetría. Existen diferentes maniobras y posturas que pueden ayudar al paciente a deglutir y minimizar el riesgo de complicaciones (44). Por ejemplo, la rotación de la cabeza hacia el lado faríngeo paralizado dirige la comida hacia el lado sano e incrementa la eficiencia del tránsito faríngeo y facilita la apertura del esfínter esofágico superior. La flexión anterior del cuello protege la vía respiratoria, la flexión posterior facilita el drenaje gravitacional faríngeo y mejora la velocidad del tránsito oral (45). GRADE modificado 1B. Recomendación fuerte, evidencia moderada calidad.

Técnicas de rehabilitación miofuncional y estimulación eléctrica

Son intervenciones que realizan los terapeutas y los fonoaudiólogos, en las cuales, generalmente, usan ejercicios que involucran los músculos de los labios, las mejillas, la lengua, el paladar blando, la faringe y la laringe. Están compuestas por ejercicios de protrusión y retrusión labial, chasquidos salientes y retruidos, estiramiento de labios, protuberancia encendida sostenida, contrarresistencia labial, oclusión y lateralización de labios hacia los lados, protrusión y retrusión de la lengua, lateralización de la lengua, empuje de lengua en contrarresistencia anterior y lateral, elevación y descenso exagerados del dorso de la lengua, etc. Se ha observado que la asociación con técnicas vocales son más efectivas para la rehabilitación de la deglución que los ejercicios miofuncionales individuales, sobre todo en pacientes con disfagia orofaríngea posterior a accidentes cerebrovasculares $(46,47)$.
Se han utilizado técnicas de estimulación térmica en áreas de la cavidad oral que contienen los receptores sensoriales involucrados en la activación de la deglución faríngea, y se ha encontrado que el frío es uno de los mejores estímulos para provocar la deglución. De ahí que se hayan propuesto estas técnicas para aumentar la conciencia sensorial en la boca antes de tragar y para reducir el retraso entre las fases oral y faríngea (aumentar la velocidad de deglución) $(48,49)$. La estimulación magnética transcraneal es una técnica segura y no invasiva, capaz de proporcionar información sobre las propiedades neurofisiológicas de un sistema objetivo y se ha utilizado con éxito para estudiar la deglución (50). En la estimulación transcraneal de corriente continua, considerada una técnica de neurorrehabilitación para disfagia, los efectos dependen de la combinación de parámetros como la intensidad actual, la duración de la estimulación y el montaje del electrodo. Generalmente, al igual que la estimulación transcraneal, es segura y bien tolerada, aun cuando es tema de investigación en pacientes con disfagia orofaríngea $(51,52,53,54)$.

\section{Consideraciones especiales de la disfagia en pacientes con demencia}

La demencia es una condición en la que existe un deterioro progresivo en la cognición que afecta la función diaria. En el 2001, la prevalencia de demencia era de aproximadamente 24 millones en todo el mundo. Se estimó que esto aumentaría a 42,3 millones en el 2020 (55). La disfagia puede desarrollarse en pacientes con demencia durante el curso de la enfermedad y, a menudo, complica su curso. Además, se ha demostrado que la disfagia puede presentarse en diferentes tipos de demencia $(56,57,58)$. Un metanálisis realizado por Alagiakrishnan et al. (16) encontró que la prevalencia de disfagia varía en los diferentes tipos de demencia y se observa con mayor frecuencia en la población de ancianos que vive en hogares geriátricos.

Los mecanismos por los cuales ocurren dificultades para deglutir varían con los 
diferentes tipos de demencia. En pacientes con enfermedad de Alzheimer se pueden observar alteraciones en los componentes sensoriales de la deglución, lo que conduce a un retraso en el tiempo de tránsito oral (56). También se han descrito disminución de la oxigenación en áreas de la corteza cerebral que comprenden muchas áreas de la red de deglución en pacientes con enfermedad de Alzheimer en estadio leve (59). En pacientes con demencia debido a enfermedad vascular, el aspecto motor de la deglución se ve afectado, lo que resulta en dificultades con la formación de bolo y la masticación (60). Los signos extrapiramidales, la disfunción autonómica y las fluctuaciones en el estado de conciencia pueden favorecer la presencia de alteraciones de la deglución en pacientes con demencia por cuerpos de Lewy o demencia por enfermedad de Parkinson (61). En la degeneración frontotemporal hay una tendencia a comer de forma compulsiva y rápida, lo que genera bolos de alimento de gran tamaño. Además, existe la tendencia a presentar episodios de filtración de alimentos a la faringe, debido quizás a la falta de conciencia de la presencia de alimentos en la boca, y se han descrito episodios de eliminación incompleta del bolo, debido a una disminución de la fuerza de contracción de la lengua, la faringe y la laringe $(57,62)$.

El tratamiento de la disfagia en la demencia es complejo, debido a que pacientes en estadios moderados y severos pueden tener dificultad para seguir instrucciones específicas y para ejecutar el conocimiento en la vida diaria (30). Se deben implementar las medidas de posicionamiento para la deglución en personas con disfagia, acompañamiento y vigilancia al comer. Según las últimas actualizaciones de las guías de la Sociedad Estadounidense de Nutrición Clínica y Metabolismo (ESPEN), la colocación de sondas nasogástricas no previene la neumonía aspirativa en pacientes con demencia, no mejora el curso de la enfermedad y, por lo tanto, no se recomienda en pacientes con demencia avanzada $(63,64$, 65). GRADE modificada: 1A. Recomendación fuerte, evidencia de alta calidad.

Una revisión Cochrane que buscaba evaluar el resultado de la nutrición por sonda enteral en ancianos con demencia avanzada y disfagia, encontró que a pesar del gran número de pacientes que recibe esta intervención, no hay pruebas suficientes para sugerir que la alimentación por sonda enteral sea beneficiosa en pacientes con demencia avanzada. Se consideró, además, que los estudios analizados eran de baja calidad, lo que genera controversia (66).

La ESPEN publicó una directriz sobre el uso de sonda nasogástrica o gastrostomía en pacientes con demencia avanzada que proporciona una lista de verificación y un algoritmo que se recomienda consultar antes de tomar la decisión de usar cualquier dispositivo de alimentación enteral a largo plazo en pacientes con demencia avanzada (65). GRADE modificado: 1A. Recomendación fuerte, evidencia de alta calidad.

Dentro de estas, las siguientes son indicaciones respecto al uso de vías alternas de alimentación en pacientes con demencia, con sus correspondientes evidencias:

Se sugiere alimentación por sonda de manera temporal en pacientes con demencia leve o moderada, si la baja ingesta nutricional es causada predominantemente por una condición potencialmente reversible. GRADE modificado: 2C. Recomendación débil, evidencia de baja calidad.

No iniciar la alimentación por sonda en pacientes con demencia severa. GRADE modificado: 1A. Recomendación fuerte, evidencia de alta calidad.

Se sugiere usar nutrición parenteral en caso de contraindicación de uso de sonda y estos líquidos parenterales deben usarse durante un tiempo limitado y se deben suspender una vez superado el periodo crítico. GRADE modificado: 2C. Recomendación débil, evidencia de baja calidad.

No se recomienda el uso de nutrición artificial (nutrición enteral, nutrición y fluidos parenterales) en la fase terminal de vida. GRADE modificado: 2A. Recomendación débil, evidencia de alta calidad.

Las intervenciones para disfagia deben iniciar de forma temprana y debe tener en cuenta los aspectos cognitivos de la alimentación. Se puede 
recomendar la adaptación de las consistencias de las comidas si el paciente y el cuidador lo aceptan (67).

\section{Conclusiones}

La disfagia orofaríngea debe ser reconocida por el personal de salud como un síndrome geriátrico que implica graves consecuencias para la salud, que incluyen malnutrición, deshidratación, infecciones respiratorias de etiología aspirativa, reingresos hospitalarios y declinación funcional hospitalaria que aceleran el proceso de fragilidad y sarcopenia y generan un círculo vicioso de disfuncionalidad, que en los ancianos se traduce a mayores costos hospitalarios y mayor morbimortalidad. Se requieren más estudios que establezcan otras evidencias de las intervenciones en los ancianos, así como entrenar al personal de salud de atención primaria para su detección e intervención precoz, debido a que consideramos que el desafío futuro implica aumentar la visibilidad de la disfagia orofaríngea e implementar medidas de intervención precoces que disminuyan de forma significativa las complicaciones y el pronóstico de las personas mayores. Faltan aún más investigaciones que integren el diagnóstico y el manejo de disfagia en individuos con demencia.

\section{Referencias}

1. Warnecke T, Dziewas R, Wirth R, Bauer JM, Prell T. Dysphagia from a neurogeriatric point of view: Pathogenesis, diagnosis and management. Z Gerontol Geriatr. 2019 Jul;52(4):330-5.

2. Khan A, Carmona R, Traube M. Dysphagia in the elderly. Clin Geriatr Med. 2014 Feb;30(1):43-53.

3. Cook IJ. Oropharyngeal dysphagia. Gastroenterol Clin North Am. 2009 Sep;38(3):411-31.

4. Takeuchi K, Aida J, Ito K, Furuta M, Yamashita Y, Osaka K.
Nutritional status and dysphagia risk among community-dwelling frail older adults. J Nutr Health Aging. 2014 Apr;18(4):352-7.

5. Wirth R, Pourhassan M, Streicher M, Hiesmayr M, Schindler K, Sieber $\mathrm{CC}$, et al. The impact of dysphagia on mortality of nursing home residents: results from the Nutrition Day Project. J Am Med Dir Assoc. 2018 Sep;19(9):775-8.

6. Madhavan A, LaGorio LA, Crary MA, Dahl WJ, Carnaby GD. Prevalence of and risk factors for dysphagia in the community dwelling elderly: a systematic review. J Nutr Health Aging. 2016;20(8):806-15.

7. Chen P-H, Golub JS, Hapner ER, Johns MM 3rd. Prevalence of perceived dysphagia and quality-of-life impairment in a geriatric population. Dysphagia. 2009 Mar;24(1):1-6.

8. Eglseer D, Halfens RJG, Schols JMGA, Lohrmann C. Dysphagia in hospitalized older patients: associated factors and nutritional interventions. J Nutr Health Aging. 2018;22(1):103-10.

9. Frías Ordóñez JS, Martínez Marín JD. Características de la disfagia en pacientes de un centro de gastroenterología en Bogotá D. C., Colombia. Rev Colomb Gastroenterol [internet]. 2018;33(44):372-8. Disponible en: htt ps://revistagastrocol.com/index.php/rc g/article/view/212/270

10. Geeganage C, Beavan J, Ellender S, Bath PMW. Interventions for dysphagia and nutritional support in acute and subacute stroke. Cochrane database Syst Rev. 2012 Oct;10:CD000323.

11. Runions S, Rodrigue N, White C. Practice on an acute stroke unit after implementation of a decision-making algorithm for dietary management 
of dysphagia. J Neurosci Nurs J Am Assoc Neurosci Nurses. 2004 Aug;36(4):200-7.

12. Ickenstein GW, Stein J, Ambrosi D, Goldstein R, Horn M, Bogdahn U. Predictors of survival after severe dysphagic stroke. J Neurol. 2005 Dec;252(12):1510-6.

13. Frattali CM, Sonies BC, ChiFishman G, Litvan I. Effects of physostigmine on swallowing and oral motor functions in patients with progressive supranuclear palsy: A pilot study. Dysphagia. 1999;14(3):165-8.

14. Affoo RH, Foley N, Rosenbek J, Shoemaker JK, Martin RE. Swallowing dysfunction and autonomic nervous system dysfunction in Alzheimer's disease: a scoping review of the evidence. J Am Geriatr Soc. 2013 Dec;61(12):2203-13.

15. Boccardi V, Ruggiero C, Patriti A, Marano L. Diagnostic assessment and management of dysphagia in patients with Alzheimer's disease. J Alzheimers Dis. 2016;50(4):947-55.

16. Alagiakrishnan $\mathrm{K}$, Bhanji RA, Kurian M. Evaluation and management of oropharyngeal dysphagia in different types of dementia: a systematic review. Arch Gerontol Geriatr. 2013;56(1):1-9.

17. Sua\#rez Escudero JC, Rueda Vallejo ZV, Orozco AF. Disfagia y neurologi\#a: iuna unio\#n indefectible? Acta Neurol Colomb [internet]. 2018;34:92-100. Disponible en: http://www.scielo.org.co/pdf/anco/ v34n1/0120-8748-anco-34-01-00092. pdf

18. Lawal A, Shaker R. Esophageal dysphagia. Phys Med Rehabil Clin. 2008 Nov 1;19(4):729-45. https://doi.o rg/10.1016/j.pmr.2008.07.003

19. Rommel N, Hamdy S. Oropharyngeal dysphagia: manifestations and diagnosis. Nat Rev Gastroenterol Hepatol. 2016 Jan;13(1):49-59.

20. Tulunay-Ugur OE, Eibling D. Geriatric dysphagia. Clin Geriatr Med. 2018 May 1;34(2):183-9. https://doi.or g/10.1016/j.cger.2018.01.007

21. Nawaz S, Tulunay-Ugur OE. Dysphagia in the older patient. Otolaryngol Clin North Am [internet]. 2018 Aug;51(4):769-77. Disponible en: https://inkinghub.elsevier.com/ret rieve/pii/S0030666518300537

22. Liu LWC, Andrews CN, Armstrong $\mathrm{D}$, Diamant $\mathrm{N}$, Jaffer $\mathrm{N}$, Lazarescu A, et al. Clinical practice guidelines for the assessment of uninvestigated esophageal dysphagia. J Can Assoc Gastroenterol. 2018 Feb 9;1(1):5-19. h ttps://doi.org/10.1093/jcag/gwx008

23. Easterling CS, Robbins E. Dementia and dysphagia. Geriatr Nurs (Minneap) [internet]. 2008;29(4):275-85. Disponible en: htt p://www.sciencedirect.com/science/art icle/pii/S0197457207003242

24. Logemann JA, Veis S, Colangelo L. A screening procedure for oropharyngeal dysphagia. Dysphagia. 1999;14(1):44-51. https://doi.org/10.1 007/PL00009583

25. Clavé P, Arreola V, Romea M, Medina L, Palomera E, Serra-Prat M. Accuracy of the volume-viscosity swallow test for clinical screening of oropharyngeal dysphagia and aspiration. Clin Nutr [internet]. 2008;27(6):806-15. Disponible en: http://www.sciencedire ct.com/science/article/pii/S026156140 8001209

26. Kertscher B, Speyer R, Palmieri M, Plant C. Bedside screening to detect oropharyngeal dysphagia in patients with neurological disorders: an updated systematic review. Dysphagia. 
2014;29(2):204-12. https://doi.org/10. 1007/s00455-013-9490-9

27. Ortega O, Martín A, Clavé P. Diagnosis and management of oropharyngeal dysphagia among older persons, state of the art. J Am Med Dir Assoc. 2017 Jul;18(7):576-82.

28. American Speech-LanguageHearing Association. Clinical indicators for instrumental assessment of dysphagia. 2000. https://doi.org/10.1 044/policy.GL2000-00047

29. O'Rourke F, Vickers K, Upton C, Chan D. Swallowing and oropharyngeal dysphagia. Clin Med. 2014 Apr;14(2):196-9.

30. Wirth R, Dziewas R, Beck AM, Clavé P, Hamdy S, Heppner HJ, et al. Oropharyngeal dysphagia in older persons - from pathophysiology to adequate intervention: a review and summary of an international expert meeting. Clin Interv Aging. 2016;11:189-208.

31. Swan K, Cordier R, Brown T, Speyer R. Psychometric properties of visuoperceptual measures of videofluoroscopic and fibreendoscopic evaluations of swallowing: a systematic review. Dysphagia. 2019 Feb;34(1):2-33.

32. Langmore SE, Schatz K, Olson $\mathrm{N}$. Endoscopic and videofluoroscopic evaluations of swallowing and aspiration. Ann Otol Rhinol Laryngol. 1991 Aug;100(8):678-81.

33. Langmore SE, Schatz K, Olsen N. Fiberoptic endoscopic examination of swallowing safety: a new procedure. Dysphagia. 1988;2(4):216-9.

34. Giraldo-Cadavid LF, LealLeaño LR, León-Basantes GA, Bastidas AR, García R, Ovalle $\mathrm{S}$, et al. Accuracy of endoscopic and videofluoroscopic evaluations of swallowing for oropharyngeal dysphagia. Laryngoscope. 2017 Sep;127(9):2002-10.

35. Martín A, Ortega O, Clavé P. Disfagia orofaríngea, un nuevo síndrome geriátrico. Rev Esp Geriatr Gerontol [internet]. 2018 Jan;53(1):3-5. Disponible en: https://linkinghub.elsevier.com/ret rieve/pii/S0211139X17302093

36. Lancaster J. Dysphagia: its nature, assessment and management. Br J Community Nurs. 2015;Suppl Nutr:S28-32.

37. Rofes L, Arreola V, Mukherjee R, Swanson J, Clavé P. The effects of a xanthan gum-based thickener on the swallowing function of patients with dysphagia. Aliment Pharmacol Ther. 2014 May 1;39(10):1169-79. https://do i.org/10.1111/apt.12696

38. Bhattacharyya N, Kotz T, Shapiro $\mathrm{J}$. The effect of bolus consistency on dysphagia in unilateral vocal cord paralysis. Otolaryngol Neck Surg Off J Am Acad Otolaryngol Neck Surg. 2003 Dec;129(6):632-6.

39. Rofes L, Arreola V, Romea M, Palomera E, Almirall J, Cabré M, et al. Pathophysiology of oropharyngeal dysphagia in the frail elderly. Neurogastroenterol Motil. 2010 Aug 1;22(8):851-e230. https://doi.org/10.1 111/j.1365-2982.2010.01521.x

40. Sura L, Madhavan A, Carnaby G, Crary MA. Dysphagia in the elderly: management and nutritional considerations. Clin Interv Aging. 2012;7:287-98.

41. Rosenvinge SK, Starke ID. Improving care for patients with dysphagia. Age Ageing. 2005 Nov 1;34(6):587-93. https://doi.org/10.109 3/ageing/afi187

42. Gallegos C, Brito-de la Fuente E, Clavé P, Costa A, Assegehegn G. Nutritional aspects of dysphagia 
management. Adv Food Nutr Res. 2017;81:271-318.

43. Potack JZ, Chokhavatia S. Complications of and controversies associated with percutaneous endoscopic gastrostomy: report of a case and literature review. Medscape J Med. 2008 Jun;10(6):142.

44. Baijens LW, Clavé P, Cras P, Ekberg O, Forster A, Kolb GF, et al. European Society for Swallowing Disorders European Union Geriatric Medicine Society white paper: oropharyngeal dysphagia as a geriatric syndrome. Clin Interv Aging. 2016;11:1403-28.

45. Cantemir S, Laubert A. [Diagnosis and treatment of dysphagia]. HNO. 2017 Apr;65(4):347-56.

46. Fraga BF de, Almeida ST de, Santana MG, Cassol M. Efficacy of myofunctional therapy associated with voice therapy in the rehabilitation of neurogenic oropharyngeal dysphagia: a pilot study. Int Arch Otorhinolaryngol. 2018 Jul;22(3):225-30.

47. Ferreira TS, Mangilli LD, Sassi FC, Fortunato-Tavares T, Limongi SCO, Andrade CRF de. Speech and myofunctional exercise physiology: a critical review of the literature. J Soc Bras Fonoaudiol. 2011 Sep;23(3):288-96.

48. Kaatzke-McDonald MN, Post E, Davis PJ. The effects of cold, touch, and chemical stimulation of the anterior faucial pillar on human swallowing. Dysphagia. 1996;11(3):198-206.

49. Rosenbek JC, Roecker EB, Wood JL, Robbins J. Thermal application reduces the duration of stage transition in dysphagia after stroke. Dysphagia. 1996;11(4):225-33.

50. Michou E, Hamdy S. Cortical input in control of swallowing. Curr Opin Otolaryngol Head Neck Surg. 2009 Jun;17(3):166-71.
51. Kumar S, Wagner CW, Frayne C, Zhu L, Selim M, Feng W, et al. Noninvasive brain stimulation may improve stroke-related dysphagia: a pilot study. Stroke. 2011 Apr;42 (4):1035-40.

52. Yang EJ, Baek S-R, Shin J, Lim JY, Jang HJ, Kim YK, et al. Effects of transcranial direct current stimulation (tDCS) on post-stroke dysphagia. Restor Neurol Neurosci. 2012;30(4):303-11.

53. Adeyemo BO, Simis M, Macea DD, Fregni F. Systematic review of parameters of stimulation, clinical trial design characteristics, and motor outcomes in non-invasive brain stimulation in stroke. Front Psychiatry. 2012;3:88.

54. Shigematsu T, Fujishima I, Ohno K. Transcranial direct current stimulation improves swallowing function in stroke patients. Neurorehabil Neural Repair. 2013 May;27(4):363-9.

55. Ferri CP, Prince M, Brayne C, Brodaty $\mathrm{H}$, Fratiglioni L, Ganguli $M$, et al. Global prevalence of dementia: a Delphi consensus study. Lancet [internet]. 2005;366(9503):2112-7. Disponible en: http://www.sciencedirect.com/scie nce/article/pii/S0140673605678890

56. Suh MK, Kim H, Na DL. Dysphagia in patients with dementia: Alzheimer versus vascular. Alzheimer Dis Assoc Disord. 2009;23 (2):178-84. https://doi.org/10. 1097/WAD.0b013e318192a539

57. Langmore SE, Olney RK, LomenHoerth C, Miller BL. Dysphagia in patients with frontotemporal lobar dementia. Arch Neurol. 2007;64(1):58-62. https://doi.org/10.1 007/PL00009583

58. Bine JE, Frank EM, McDade HL. Dysphagia and dementia in subjects 
with Parkinson's disease. Dysphagia. 1995;10(3):160-4. https://doi.org/10.1 007/BF00260970

59. Humbert IA, McLaren DG, Kosmatka K, Fitzgerald M, Johnson S, Porcaro E, et al. Early deficits in cortical control of swallowing in Alzheimer's disease. J Alzheimers Dis. 2010;19(4):1185-97.

60. Edahiro A, Hirano H, Yamada R, Chiba Y, Watanabe Y. [Comparative study of eating behavior in elderly patients with Alzheimer's disease and vascular dementia: a first report. - Comparison of disturbed eating behavior - ]. Nihon Ronen Igakkai Zasshi. 2013;50(5):651-60.

61. Shinagawa S, Adachi $\mathrm{H}$, Toyota Y, Mori T, Matsumoto I, Fukuhara R, et al. Characteristics of eating and swallowing problems in patients who have dementia with Lewy bodies. Int Psychogeriatrics. 2009;21(3):520-5. https://doi.org/10.1 017/S1041610209008631

62. Lewis C, Walterfang M, Velakoulis D, Vogel AP. A review: mealtime difficulties following frontotemporal lobar degeneration. Dement Geriatr Cogn Disord. 2018;46(5-6):285-97.

63. Volkert D, Beck AM, Cederholm T, Cruz-Jentoft A, Goisser S, Hooper $\mathrm{L}$, et al. ESPEN guideline on clinical nutrition and hydration in geriatrics. Vol. 38: Clinical Nutrition. Philadelphia: Elsevier; 2019. p. 10-47.

64. Volkert D, Berner YN, Berry E, Cederholm T, Coti Bertrand P, Milne $A$, et al. ESPEN guidelines on enteral nutrition: geriatrics. Clin Nutr. 2006 Apr;25(2):330-60.

65. Volkert D, Chourdakis M, FaxenIrving $G$, Frühwald $T$, Landi $F$, Suominen MH, et al. ESPEN guidelines on nutrition in dementia. Clin Nutr. 2015 Dec;34(6):1052-73.
66. Sampson EL, Candy B, Jones L. Enteral tube feeding for older people with advanced dementia. Cochrane database Syst Rev. 2009 Apr;2009(2):CD007209.

67. Rösler A, Pfeil S, Lessmann H, Höder J, Befahr A, von Renteln-Kruse W. Dysphagia in dementia: influence of dementia severity and food texture on the prevalence of aspiration and latency to swallow in hospitalized geriatric patients. J Am Med Dir Assoc. 2015 Aug;16(8):697-701.

\section{Notas}

Conflicto de intereses Los autores declaran no tener conflictos de intereses para esta revisión. 